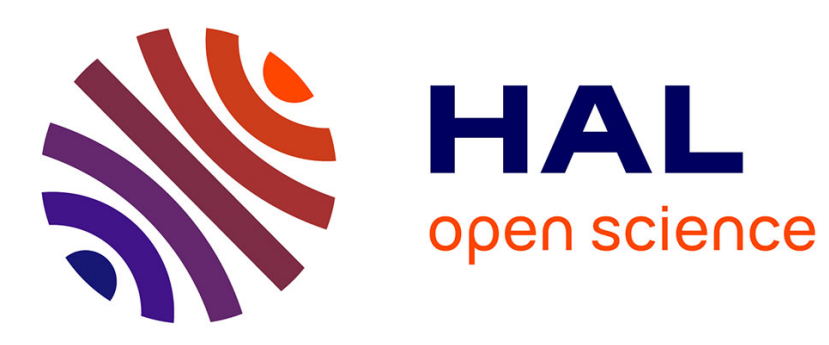

\title{
Index Modulation Aided Uplink NOMA for Massive Machine Type Communications
}

Muhammad Basit Shahab, Sarah Johnson, Mahyar Shirvanimoghaddam, Marwa Chafii, Ertugrul Basar, Mischa Dohler, Muhammad Basit Shahab

\section{- To cite this version:}

Muhammad Basit Shahab, Sarah Johnson, Mahyar Shirvanimoghaddam, Marwa Chafii, Ertugrul Basar, et al.. Index Modulation Aided Uplink NOMA for Massive Machine Type Communications. IEEE Wireless Communications Letters, 2020, 9 (12), pp.2159-2162. 10.1109/LWC.2020.3015920 . hal-03210889

\section{HAL Id: hal-03210889 \\ https://hal.science/hal-03210889}

Submitted on 28 Apr 2021

HAL is a multi-disciplinary open access archive for the deposit and dissemination of scientific research documents, whether they are published or not. The documents may come from teaching and research institutions in France or abroad, or from public or private research centers.
L'archive ouverte pluridisciplinaire HAL, est destinée au dépôt et à la diffusion de documents scientifiques de niveau recherche, publiés ou non, émanant des établissements d'enseignement et de recherche français ou étrangers, des laboratoires publics ou privés. 


\title{
Index Modulation Aided Uplink NOMA for Massive Machine Type Communications
}

\author{
Muhammad Basit Shahab, Member, IEEE, Sarah J. Johnson, Member, IEEE, Mahyar Shirvanimoghaddam, Senior \\ Member, IEEE, Marwa Chafii, Member, IEEE, Ertugrul Basar, Senior Member, IEEE, and Mischa \\ Dohler, Fellow, IEEE
}

\begin{abstract}
Single-carrier frequency-division multiple access (SC-FDMA) based index modulation (IM), referred here as SC-IM, allows users to implicitly transmit a part of their data bits by switching on some of their allocated subcarriers, followed by $M$-ary constellation symbols transmission over the activated subcarriers. This sparse use of subcarriers provides better energy efficiency and error rate. Using these benefits, this article exploits SC-IM with uplink power domain non-orthogonal multiple access (NOMA), where all users with the same allocated subcarrier set can simultaneously transmit their SC-IM data with different power levels, which are exploited by the receiver to perform multi-user detection. Performance of the proposed technique is analyzed and compared with conventional NOMA. It is shown that the proposed scheme can achieve energy efficient massive connectivity, which is a primary requirement for massive machine-type communications.
\end{abstract}

Index Terms-Index modulation (IM), non-orthogonal multiple access (NOMA), single-carrier frequency-division multiple access (SC-FDMA), massive machine-type communications (mMTC).

\section{INTRODUCTION}

$\mathbf{M}$ ASSIVE machine-type communications (mMTC) provides the framework to enable connectivity of a huge number of IoT (Internet of Things) devices with little or no human intervention. Different from the conventional humantype communications (HTC), mMTC features mainly uplink transmissions of small-sized data from a huge number of IoT devices with high energy efficiency demands [1], [2]. This requires a dramatic paradigm shift from the communication techniques mostly designed for HTC.

Index modulation (IM) is an energy/spectral efficient technique, where some part of the information bits is implicitly encoded in the indices of the used system resources [3]. In [4],

Manuscript received March xx, 2020; revised June xx, 2020; accepted August xx, 2020. Date of publication XXXX. This research was supported by the Australian Research Council under the Discovery Projects grant DP180100606. (Corresponding author: Muhammad Basit Shahab.)

Muhammad Basit Shahab and Sarah J. Johnson are with the School of Electrical Engineering and Computing, University of Newcastle, Callaghan NSW 2308, Australia (e-mail: basit.shahab@newcastle.edu.au; sarah.johnson@newcastle.edu.au).

Mahyar Shirvanimoghaddam is with the School of Electrical and Information Engineering, University of Sydney, Camperdown NSW 2006, Australia (email: mahyar.shirvanimoghaddam@sydney.edu.au).

Marwa Chafii is with ETIS, UMR 8051, CY Cergy Paris Université, ENSEA, CNRS, 95000 Cergy, France, Email: marwa.chafii@ensea.fr.

Ertugrul Basar is with the Department of Electrical and Electronics Engineering, Koç University, Sariyer 34450, Istanbul, Turkey (email: ebasar@ku.edu.tr).

Mischa Dohler is with the Department of Informatics, King's College London, London WC2B 4BG, U.K (email: mischa.dohler@kcl.ac.uk).
IM is applied to orthogonal frequency division multiplexing (OFDM) to enhance the spectral efficiency, where a subset of subcarriers are activated and their indices are used to convey information besides constellation symbols. However, the technique suffers from high peak to average power ratio inherent to OFDM. To this end, a single-carrier frequency division multiple access (SC-FDMA) based IM (SC-IM) technique is proposed in [5], [6]. Considering its 50\% energy efficiency compared to SC-FDMA [5], SC-IM is considered in this work to satisfy the low energy requirements of mMTC.

While SC-IM demonstrates energy efficiency gains, the scheme in [5], [6] is still orthogonal multiple access (OMA) based, where a set of subcarriers is dedicated to one user. However, by considering the huge number of devices in mMTC, power domain non-orthogonal multiple access (NOMA) with SC-IM is considered in this work. NOMA allows multiple users to transmit data over the same radio resources with different power levels, and using successive interference cancellation (SIC) for multi-user detection (MUD) at the receiver, hence opening ways for massive connectivity over limited resources [7]. Moreover, there exists a consensus between academia and industry that NOMA schemes should be investigated for diverse $5 \mathrm{G}$ usage scenarios, and $5 \mathrm{G}$ should support uplink NOMA at least for mMTC [8], [9].

Owing to the benefits of different NOMA and IM schemes, their integration has attracted much interest in recent years [10], [11], and is the motivation behind this work. The proposed technique, referred to as SC-IM-NOMA, allows users to transmit their data through SC-IM (subcarrier activation and $M$-ary constellation), while massive access is achieved through NOMA. For MUD, two detectors, i.e., SIC and joint maximum likelihood (JML), are proposed. Performance of SC-IM-NOMA is analyzed and compared with classical SCFDMA NOMA (SC-NOMA) to show the achieved gains.

\section{System Model And TRAnsmission PRotocol}

\section{A. System Model}

Consider an uplink scenario, where two $(L=2)$ users, $l \in\{1,2\}=\left\{\mathrm{UE}_{1}, \mathrm{UE}_{2}\right\}$, transmit their messages to the base station (BS) over the same resource block (RB, a set of adjacent subcarriers). The $\mathrm{UE}_{1} \rightarrow \mathrm{BS}$ and $\mathrm{UE}_{2} \rightarrow \mathrm{BS}$ channel gains are represented as $\left|h_{1}\right|^{2}$ and $\left|h_{2}\right|^{2}$ respectively, such that $\left|h_{1}\right|^{2}>\left|h_{2}\right|^{2}$. Both the users transmit their SCIM based message signals to the BS with powers $P_{1}$ and $P_{2}$ respectively, such that their received signal powers at the BS are in the order $P_{1}^{r}>P_{2}^{r}$. 


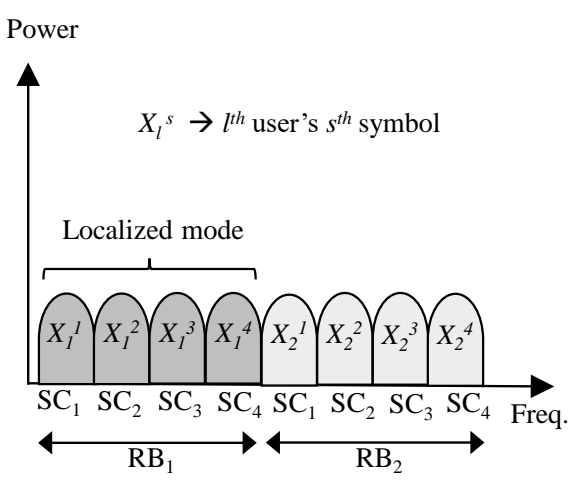

(a) SC-FDMA

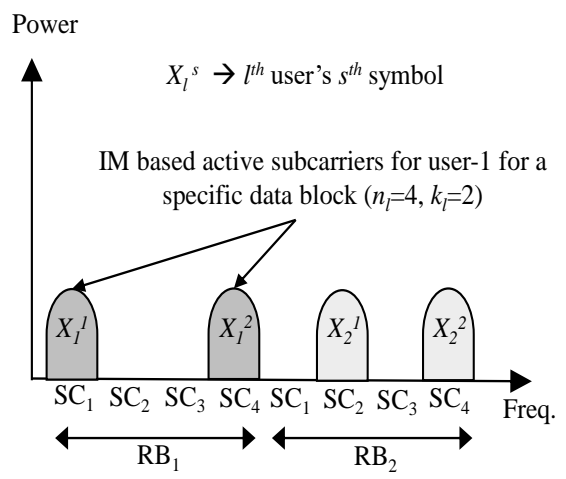

(b) SC-IM

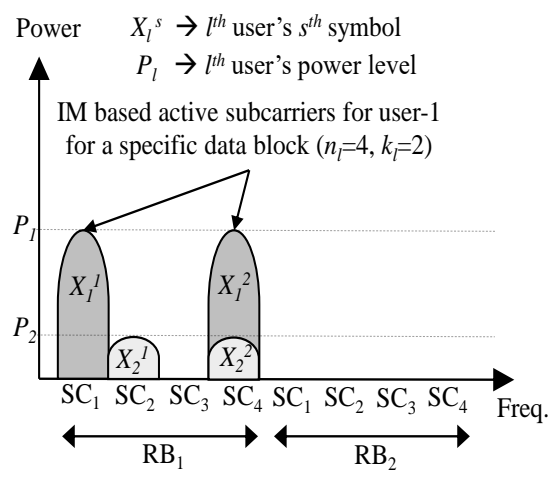

(c) SC-IM-NOMA

Fig. 1: Illustration of subcarrier allocation among $(L=2)$ users; (a) SC-FDMA with localized allocation for $n_{l}=4$, (b) SC-IM using $n_{l}=4$ and $k_{l}=2$, and (c) SC-IM-NOMA.

\section{B. Conventional SC-IM}

Consider a $L=2$ users scenario, where each $l^{\text {th }}$ user has $b_{l}$ bits to transmit in each data block. Moreover, $n_{l}$ and $k_{l}$ represent its total allocated and active subcarriers respectively. The data stream of $b_{l}=b_{l}^{\text {ind }}+b_{l}^{\text {sym }}$ bits is transmitted in each SC-IM block, where the $b_{l}^{\text {ind }}=\left\lfloor\log _{2}\left(\begin{array}{l}n_{l} \\ k_{l}\end{array}\right)\right\rfloor$ bits determine the $k_{l}$ active subcarriers indices, and $b_{l}^{\mathrm{sym}}=k_{l} \log _{2}(M)$ determine the set of $M$-ary modulated symbols to be transmitted using the $k_{l}$ active subcarriers. The total number of transmitted bits of an $l^{\text {th }}$ user in a SC-IM block can then be written as $b_{l}=$ $b_{l}^{\text {ind }}+b_{l}^{\text {sym }}=\left|\log _{2}\left(\begin{array}{c}n_{l} \\ k_{l}\end{array}\right)\right|+k_{l} \log _{2}(M)$ [5], [6].

A transmitter model for conventional SC-IM is given in [6, Fig. 1a]. The incoming bits of an $l^{t h}$ user are fed to the IM block, that generates an output vector of the form $\mathbf{x}_{l}=\left[\begin{array}{llll}x_{l}^{1} & 0 & 0 & x_{l}^{2}\end{array}\right]^{T}$, consisting of symbols from a pre-defined constellation and zeros associated to the inactive subcarriers, where $x_{l}^{s}$ represents the $s^{t h}$ constellation symbol of $l^{\text {th }}$ user. Different index selection rules can be adopted in this context. For instance, if $b_{l}^{\text {ind }}=2, n_{l}=4$, and $k_{l}=2$, then an example of IM bits to active subcarrier selection can be $00 \rightarrow\{1,3\}$, $01 \rightarrow\{1,4\}, 10 \rightarrow\{2,3\}$, and $11 \rightarrow\{2,4\}$. On this output vector, a $n_{l}$-point discrete Fourier transform (DFT) is performed, followed by subcarrier mapping in order to develop an access mode of the users. This is followed by $N_{\text {FFT }}$-point inverse DFT (IDFT), cyclic prefix (CP) insertion, and finally signal transmission. An illustration of subcarrier activation and data mapping for SC-FDMA and SC-IM is shown in Figs. 1a and $1 \mathrm{~b}$ respectively. At the receiver (shown in [6, Fig. 1b]), an inverse of these processes takes place i.e., CP removal, $N_{\mathrm{FFT}^{-}}$ point DFT, subcarrier de-mapping (with additional channel equalization), $N_{l}$-point IDFT, and finally data detection.

\section{SC-IM-NOMA}

As SC-IM allocates a set of subcarriers to each user, the number of users that can be served in the system is limited. In this context, the use of NOMA with SC-IM is a very feasible choice for enabling massive connectivity. The proposed SCIM-NOMA technique takes benefits from the energy efficiency and BER improvement of SC-IM and massive connectivity from NOMA to simultaneously improve these performance metrics. Due to the sparsity of subcarrier use (courtesy of SC-IM), the interference faced by a user over a particular subcarrier due to multiple served users in NOMA will be much less than traditional SC-NOMA, where it will be the total number of multiplexed users over the subcarrier set.

An example of resource mapping in SC-IM-NOMA for the two users case is shown in Fig. 1c. Both users transmit their data over the same RB with different power levels using NOMA, whereas the symbol mapping on the subcarriers is according to SC-IM. The difference in the power levels is exploited by the BS to perform MUD. It is noticeable that interference faced by a user on each subcarrier is significantly less in SC-IM-NOMA compared to what it would be in conventional SC-NOMA. Moreover, it can be seen in Fig. 1c that both users can transmit over $\mathrm{RB}_{1}$, and therefore $\mathrm{RB}_{2}$ can be used by any other users in the system.

At the transmitter side, each $l^{\text {th }}$ user carries out the conventional SC-IM processing as in [6, Fig. 1a], but the signals from both users are transmitted with different power allocation (PA) factors which, after passing through the communication channel, reach the BS. After CP removal, $N_{\text {FFT-point DFT, }}$ subcarrier de-mapping, and $N_{l}$-point IDFT, the superimposed signal is fed to the detector. The difference between the receivers of SC-IM (in [6, Fig. 1b]) and SC-IM-NOMA lies in the fact that the signal received at detector of the later is a superimposed signal, requiring a NOMA-based MUD. This received signal over the $n_{l}$ subcarriers set can be written as

$$
\mathbf{y}=\sum_{l=1}^{L} \sqrt{P_{l}} \operatorname{diag}\left(\mathbf{h}_{l}\right) \mathbf{x}_{l}+\mathbf{w}=\operatorname{diag}(\mathbf{G X})+\mathbf{w},
$$

where $\mathbf{y}=\left[\begin{array}{lll}y(1) & \cdots & y\left(n_{l}\right)\end{array}\right]^{T}, \mathbf{h}_{l}=\left[\begin{array}{llll}h_{l}(1) & \cdots & h_{l}\left(n_{l}\right)\end{array}\right]^{T}$, $\mathbf{x}_{l}=\left[\begin{array}{llll}x_{l}(1) & \cdots & x_{l}\left(n_{l}\right)\end{array}\right]^{T}, \mathbf{G}=\left[\begin{array}{llll}\sqrt{P_{1}} \mathbf{h}_{1} & \sqrt{P_{2}} \mathbf{h}_{2} & \cdots & \sqrt{P_{L}} \mathbf{h}_{L}\end{array}\right]$, $\mathbf{X}=\left[\begin{array}{llll}\mathbf{x}_{1} & \mathbf{x}_{2} & \cdots & \mathbf{x}_{L}\end{array}\right]^{T}$, and $\mathbf{w}=\left[\begin{array}{llll}w_{1} & w_{2} & \cdots & w_{n_{l}}\end{array}\right]^{T}$, with $\operatorname{diag}($.$) and (.)^{T}$ denoting diagonal and transpose respectively.

In order to recover the sparse SC-IM transmitted vector $\mathbf{x}_{l}=$ $\left[x_{l}(1) \cdots x_{l}\left(n_{l}\right)\right]^{T}$ of each user from this superimposed signal $\mathbf{y}$, the proposed receivers are discussed next. 
1) JML detector: Given the estimated channel vector $\hat{\mathbf{h}}_{l}$ of each $l^{\text {th }}$ user, and motivated by [12], the JML detector performs an exhaustive search to simultaneously recover the transmitted vector $\hat{\mathbf{x}}_{l}$ of each user. The operation is given as

$$
\left\{\hat{\mathbf{x}}_{l}\right\}_{l=1}^{L}=\underset{\mathbf{x}_{l} \in \chi}{\arg \min }\left\|\mathbf{y}-\sum_{l=1}^{L} \sqrt{P_{l}} \operatorname{diag}\left(\hat{\mathbf{h}}_{l}\right) \mathbf{x}_{l}\right\|^{2},
$$

where $\left\{\hat{\mathbf{x}}_{l}\right\}_{l=1}^{L}$ represents the recovered vector set of each user; $L=2$ can be used for the considered two-user scenario.

JML checks $\mathbf{y}$ for all possible combinations of transmit vectors of users, which is computationally complex. However, this gives the optimum performance, which can be used as a benchmark when designing any other low complexity receiver. Moreover, due to the joint search over all possible transmit vector combinations, it may not suffer from the error floor issues common to SIC receivers as shown in Sec. III-C.

2) SIC detector: Inspired by conventional SIC in NOMA, this receiver works on detecting the signals of multiplexed users in decreasing order of their received powers. For the twouser scenario, assuming that the received signal power of $\mathrm{UE}_{1}$ is greater than $\mathrm{UE}_{2}\left(P_{1}^{r}>P_{2}^{r}\right)$, the $\mathrm{BS}$ first tries to recover the high power signal of $\mathrm{UE}_{1}$ by checking all possibilities of the transmitted vector $\mathbf{x}_{1}$, and treating the low power $\mathrm{UE}_{2}$ signal as noise. The operation can be written as

$$
\hat{\mathbf{x}}_{1}=\underset{\mathbf{x}_{1} \in \chi}{\arg \min }\left\|\mathbf{y}-\sqrt{P_{1}} \operatorname{diag}\left(\hat{\mathbf{h}}_{1}\right) \mathbf{x}_{1}\right\|^{2} .
$$

Once $\hat{\mathbf{x}}_{1}$ is recovered, the BS subtracts its impact from $\mathbf{y}$ as a step of SIC. Let the signal after SIC be denoted as $\mathbf{y}_{\text {SIC }}$. The BS then tries to recover $\mathrm{UE}_{2}$ 's transmitted vector by performing similar step, as done earlier for $\mathrm{UE}_{1}$, on $\mathbf{y}_{\mathrm{SIC}}$.

While this looks straightforward, it may cause error floors for users, typical to uplink NOMA SIC receivers as discussed in Sec. III-C. As UE ${ }_{1}$ 's transmitted signal is initially recovered by treating the low power $\mathrm{UE}_{2}$ 's signal as interference, the overall signal-to-noise-and-interference ratio of $\mathrm{UE}_{1}$ tends to saturate at high transmit powers of both users. This may cause an error floor for $\mathrm{UE}_{1}$, which is then propagated to $\mathrm{UE}_{2}$.

3) Other Detectors: Considering the error floor in SIC, and high computational complexity in JML, the design of low complexity sub-optimal receivers is an important issue. In this context, the log likelihood ratio (LLR) and sparsity detector receivers in [6] for conventional SC-IM can be extended to SCIM-NOMA. One such extension of LLR receiver for an IMNOMA scheme is designed in [13], where the LLR values for events representing the number of users activating a particular subcarrier are calculated, followed by finding the contributing users and their symbols for the most likely events. The design of such receivers for SC-IM-NOMA is a crucial issue, and is subject to our future research works.

\section{Performance Evaluation}

\section{A. Average subcarrier load}

Due to the use of SC-IM, SC-IM-NOMA experiences a lower average user load per subcarrier $(\xi)$, calculated as

$$
\xi=\sum_{l=1}^{L} \frac{k_{l}}{n_{l}} .
$$

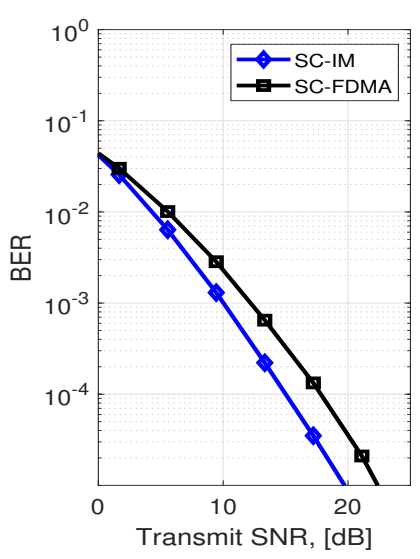

(a) SC-IM vs SC-FDMA

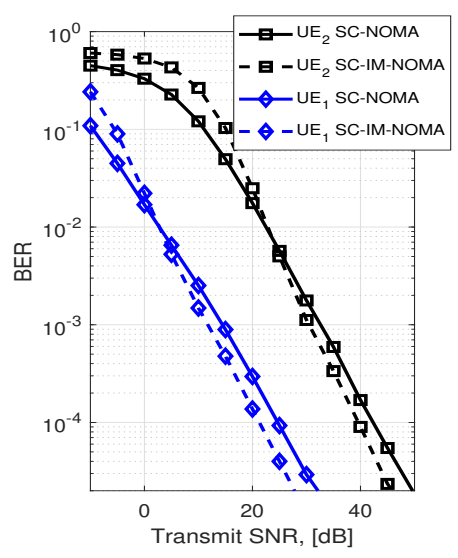

(b) SC-IM-NOMA vs SC-NOMA
Fig. 2: BER performance analysis.

Consider $L=2$ users multiplexed over $n_{l}=4$ subcarriers with different power levels. In case of SC-IM-NOMA, for $k_{l}=1,2$, and 3 , the average user data load per subcarrier will be $\xi=0.5,1.0$, and 1.5 respectively. For conventional SC-NOMA, each user's data will be mapped on all subcarriers, and $\xi$ will be same as the total number of multiplexed users i.e., 2 here. Thus, the average subcarrier load in SC-IMNOMA is comparatively lower than SC-NOMA. For simple SC-IM-OMA using time division multiple access, where users transmit in different time slots, $\xi$ will be $\frac{k_{l}}{n_{l}}$, which gives $\xi=0.25,0.5$, and 0.75 for $k_{l}=1,2$, and 3. However, as each user now transmits for $\frac{1}{L}$ time slots compared to the proposed SC-IM-NOMA, the users in SC-IM-OMA will need to use higher order modulation schemes to match the data rate of SC-IM-NOMA, degrading BER and energy efficiency.

\section{B. Energy efficiency}

The energy efficiency of the $l^{\text {th }}$ user in SC-IM-NOMA compared to SC-NOMA is calculated as

$$
\begin{aligned}
\eta_{l} & =\frac{\epsilon_{\text {SC-IM-NOMA }}-\epsilon_{\text {SC-NOMA }}}{\epsilon_{\text {SC-NOMA }}} \\
& =\frac{\alpha\left(k_{l} \log _{2}(M)-n_{l} \log _{2}(\hat{M})\right)}{\alpha n_{l} \log _{2}(\hat{M})}=\left\{\frac{k_{l} \log _{2}(M)}{n_{l} \log _{2}(\hat{M})}-1\right\} \times 100,
\end{aligned}
$$

where $\alpha$ is power level, $\epsilon$ is total bits normalized energy, and $M$ and $\hat{M}$ are the modulation sizes in SC-IM-NOMA and SCNOMA respectively to achieve the same spectral efficiency.

When $\hat{M}=2$ in SC-NOMA, 4 bits are transmitted over $n_{l}=4$ subcarriers. To achieve the same spectral efficiency, SC-IM-NOMA with $n_{l}=4$ and $k_{l}=2$ also uses $M=2$. For this case, $\eta_{l}=-50 \%$. For large values of $\hat{M}$, with $n_{l}, k_{l}$ fixed, comparatively higher values of $M$ are needed, which reduces the energy saving of SC-IM-NOMA. For instance, when $\hat{M}=16$, the value of $M=128$ to achieve same spectral efficiency with $n_{l}=4$ and $k_{l}=2$ gives $\eta_{l}=-12.5 \%$. However, such case is less probable in mMTC, where lower order modulation schemes are preferred. For SC-IM-OMA case, each user transmits only $\frac{1}{L}$ times compared to SC-IMNOMA. Let the transmit power of a user in SC-IM-OMA be 
$\beta$, and the modulation size to meet the data rate of SC-IMNOMA be $M_{o}$, then $n_{l}$ of SC-IM-NOMA compared to SCIM-OMA can be written as $\left(\frac{\alpha L \log _{2}(M)}{\beta \log _{2}\left(M_{o}\right)}-1\right) \times 100$, which depends on $\alpha, \beta, L$, and the modulation sizes.

\section{BER of users}

Initially, a simple SC-IM vs conventional SC-FDMA BER plot is shown in Fig. 2a by considering the same simulation parameters used in [5, Table I]. It can be seen that the BER performance of SC-IM is better than that of SC-FDMA.

Next, we compare the BER of SC-IM-NOMA using JML (optimal) receiver with SC-NOMA for a $L=2$ users scenario under Rayleigh fading channel in Fig. 2b. The $\mathrm{UE}_{1} \rightarrow \mathrm{BS}$ and $\mathrm{UE}_{2} \rightarrow \mathrm{BS}$ normalized distances are $d_{1}=0.5$ and $d_{2}=1.0$ respectively. Normalized transmit powers of $\mathrm{UE}_{1}\left(P_{1}\right)$ and $\mathrm{UE}_{2}\left(P_{2}\right)$ are set as 1 and 0.5 respectively i.e., $P_{2}=\frac{P_{1}}{2}$. It is worth noting that the constraint of $P_{1}+P_{2} \leq 1$, normally applied in downlink NOMA scenarios, is not a necessity in the uplink case. The index mapping rule for IM is the same as discussed in Sec II-B, modulation type is BPSK, and total subcarriers are 4. Overall, it can be seen that the BER performance of $\mathrm{UE}_{1}$ in SC-IM-NOMA is better than SCNOMA, whereas the performance of $\mathrm{UE}_{2}$ is only better in the high SNR region. These trends may vary based on the system parameters. Moreover, while simulation results are shown here for SC-IM-NOMA, the upper bound on BER performance from (1) can be calculated as

$$
P_{e} \leq \frac{1}{D 2^{D}} \sum_{\mathbf{X}} \sum_{\hat{\mathbf{x}}} \operatorname{Pr}(\mathbf{X} \rightarrow \hat{\mathbf{X}}) \delta(\mathbf{X}, \hat{\mathbf{X}}),
$$

where $D=L b_{l}, \hat{\mathbf{X}}=\left[\hat{\mathbf{x}}_{1} \hat{\mathbf{x}}_{2}, \cdots, \hat{\mathbf{x}}_{L}\right]^{T}$ is the estimated data matrix, $\delta(\mathbf{X}, \hat{\mathbf{X}})$ is the bits difference count between $\mathbf{X}$ and $\hat{\mathbf{X}}$, and $\operatorname{Pr}(\mathbf{X} \rightarrow \hat{\mathbf{X}})$ is the pairwise error probability, that can be calculated by [12], but using matrix $\mathbf{G}$ in place of $\mathbf{H}$ to accommodate the NOMA power levels.

Finally, Figs. 3a and $3 \mathrm{~b}$ provide a BER performance comparison between the JML and SIC receivers for $L=2$ and $L=3$ users, respectively. It can be clearly seen that the JML outperforms the SIC detector for both cases, where the later suffers from error floors in the high SNR regime. This behavior is typical to uplink SIC-based NOMA receivers, which becomes more prominent for higher number of users. In this context, for the joint detection, it can be seen that the error floor issue is satisfactorily resolved; detailed mathematical analysis of JML compared to SIC receiver for conventional NOMA is provided in [14].

\section{CONCLUSIONS}

This letter proposes an SC-IM-NOMA scheme to simultaneously achieve the energy efficiency and BER performance gains of SC-IM, while providing massive connectivity through power domain NOMA. Performance of the proposed scheme is analyzed and compared with conventional SC-NOMA scheme to demonstrate the achieved energy and BER gains. In this context, two types of receivers are used. A detailed mathematical analysis of BER and design of low complexity receivers is subject to our future research work.

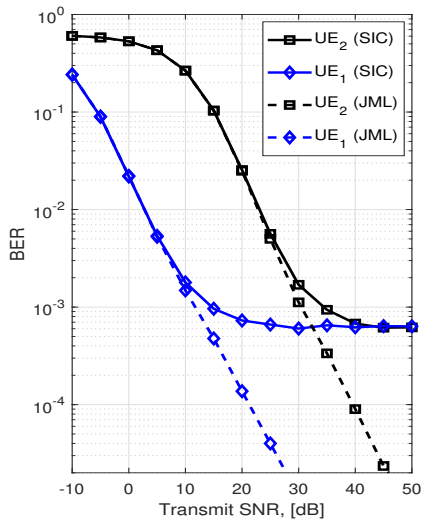

(a) JML vs SIC for $L=2$ case.

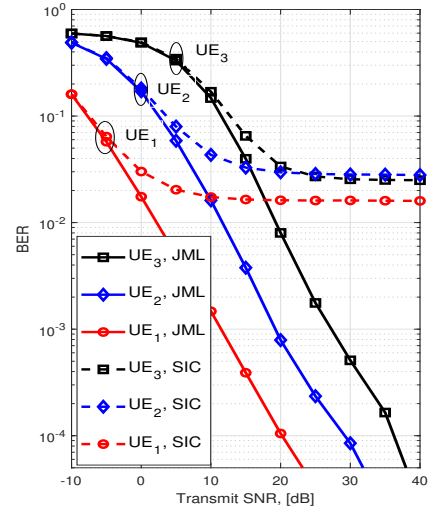

(b) JML vs SIC for $L=3$ case.
Fig. 3: BER performance analysis of SC-IM-NOMA; For $L=$ 2 case, $d_{1}=0.5, d_{2}=1.0, P_{1}=1.0$, and $P_{2}=0.5$, and for $L=3$ case, $d_{1}=0.4, d_{2}=0.75, d_{3}=1.0, P_{1}=1.0$, $P_{2}=0.7$, and $P_{3}=0.4$.

\section{REFERENCES}

[1] ITU-R, "IMT vision-framework and overall objectives of the future development of IMT for 2020 and beyond," Int. Telecommun. Union, Geneva, Switzerland, ITU Recommendation M.2083-0, Sep. 2015.

[2] P. Jain, P. Hedman, and H. Zisimopoulos, "Machine type communications in 3GPP systems," IEEE Commun. Mag., vol. 50, no. 11, pp. 28-35, Nov. 2012.

[3] X. Cheng, M. Zhang, M. Wen, and L. Yang, "Index modulation for 5G: Striving to do more with less," IEEE Wireless Commun., vol. 25, no. 2, pp. 126-132, May 2018.

[4] E. Başar, Ü. Aygölü, E. Panayırcı, and H. V. Poor, "Orthogonal frequency division multiplexing with index modulation," IEEE Trans. Signal Process., vol. 61, no. 22, pp. 5536-5549, Nov. 2013.

[5] M. Chafii, F. Bader, and J. Palicot, "SC-FDMA with index modulation for M2M and IoT uplink applications," in Proc. IEEE Wireless Commun. Netw. Conf. (WCNC), Apr. 2018, pp. 1-5.

[6] J. Manco-Vasquez, M. Chafii, and F. Bader, "Tailoring index-modulation for uplink IoT and M2M networks," in Proc. IEEE Wireless Commun. Netw. Conf. (WCNC), Apr. 2019, pp. 1-6.

[7] Y. Saito et al., "Non-orthogonal multiple access (NOMA) for cellular future radio access," in Proc. IEEE 77th Veh. Technol. Conf. (VTC), Jun. 2013, pp. 1-5.

[8] 3GPP, "Study on non-orthogonal multiple access (NOMA) for NR (release 16)," 3GPP, Valbonne, France, Technical Report 38.812 V0.2.0, Nov. 2018.

[9] M. B. Shahab, R. Abbas, M. Shirvanimoghaddam, and S. J. Johnson, "Grant-free non-orthogonal multiple access for IoT: A survey," IEEE Commun. Surveys Tuts., pp. 1-1, 2020.

[10] S. Doğan, A. Tusha, and H. Arslan, "NOMA with index modulation for uplink URLLC through grant-free access," IEEE J. Sel. Topics Signal Process., vol. 13, no. 6, pp. 1249-1257, May 2019.

[11] Q. Li, M. Wen, E. Basar, H. V. Poor, and F. Chen, "Spatial modulationaided cooperative NOMA: Performance analysis and comparative study," IEEE J. Sel. Topics Signal Process., vol. 13, no. 3, pp. 715-728, Feb. 2019.

[12] S. Althunibat, R. Mesleh, and T. F. Rahman, "A novel uplink multiple access technique based on index-modulation concept," IEEE Trans. Commun., vol. 67, no. 7, pp. 4848-4855, Apr. 2019.

[13] J. Li, Q. Li, S. Dang, M. Wen, X. Jiang, and Y. Peng, "Low-complexity detection for index modulation multiple access," IEEE Wireless Commun. Lett., vol. 9, no. 7, pp. 943-947, Feb. 2020.

[14] J. S. Yeom, H. S. Jang, K. S. Ko, and B. C. Jung, "BER performance of uplink NOMA with joint maximum-likelihood detector,' IEEE Trans. Veh. Technol., vol. 68, no. 10, pp. 10295-10300, Oct. 2019. 